\title{
Corela
}

Cognition, représentation, langage

HS-29 | 2019

Questions et exclamations au prisme de plusieurs approches linguistiques

\section{Les questions en grammaire générative}

Jean-Charles KHALIFA

\section{(2) OpenEdition}

1 Journals

Édition électronique

URL : http://journals.openedition.org/corela/8648

DOI : $10.4000 /$ corela.8648

ISSN : 1638-573X

Éditeur

Cercle linguistique du Centre et de l'Ouest - CerLICO

Référence électronique

Jean-Charles KHALIFA, «Les questions en grammaire générative », Corela [En ligne], HS-29 | 2019, mis en ligne le 05 septembre 2019, consulté le 28 octobre 2019. URL : http://journals.openedition.org/ corela/8648; DOI : 10.4000/corela.8648

Ce document a été généré automatiquement le 28 octobre 2019

\section{(c) (i) (3) (2)}

Corela - cognition, représentation, langage est mis à disposition selon les termes de la licence Creative Commons Attribution - Pas d'Utilisation Commerciale - Partage dans les Mêmes Conditions 4.0 International. 


\title{
Les questions en grammaire générative
}

\author{
Jean-Charles KHALIFA
}

\section{Introduction}

1 Pour traiter des questions en grammaire générative (GG), nous commencerons par deux remarques liminaires qui reprennent les remarques formulées au colloque «Théories linguistiques en dialogue " portant sur la prédication (Khalifa 2017 in Vincent-Durroux L. (éd.)). : d'une part - et pour bien préciser « d'où nous parlons ", comme on disait autrefois dans un certain contexte politique - on dit parfois qu'il existe autant de théories syntaxiques que de syntacticiens, mais nous prendrons ici pour cadre théorique un compromis entre plusieurs versions simplifiées tournant autour de ce que l'on nomme Principles and Parameters (P\&P, voir par exemple Culicover 1997), ce qui renvoie également, mais nous n'en dirons rien ici, derrière ce terme de «Principes ", à la notion de Grammaire Universelle (Universal Grammar, cf. inter alii Chomsky 1965), déclinée selon les langues naturelles en paramètres spécifiques.

2 En second lieu, redisons encore que le domaine de travail du syntacticien est limité, comme l'illustre la citation 1 (CIT. 1) d'un célèbre fonctionnaliste, à la phrase et ne saurait, à quelques exceptions près, être étendue à l'échelle du texte. Pour dire les choses de façon plus brutale, aucune explication de texte ne saurait prendre pour cadre théorique une théorie syntaxique quelconque. Nous allons plutôt aller chercher dans le texte qui nous a été fourni des illustrations de divers aspects du thème de ce volume, à savoir les questions. Pour cela, nous exposerons les éléments théoriques pertinents en les associant à des extraits du corpus. Nous aborderons donc le schéma de l'événement, puis les questions directes et enfin les questions imbriquées. Nous conclurons en évoquant brièvement les travaux sur la périphérie gauche de la phrase et l'analyse en Split-CP, qui font évoluer sensiblement les perspectives.

3 By and large, therefore, the chapters that follow take as their domain the traditional realm of syntax, the terrain from the sentence to the word. Grammatically, that is 
where the action is; and within that, the fundamental unit of organization is the clause.

\section{Le schéma de l'événement}

6 Rappelons, pour situer les choses de façon plus large et transthéorique, comment s'organise le schéma fondamental d'événement ${ }^{1}$ que, selon Carnie (2008), on retrouve sous une forme ou une autre dans toutes les théories syntaxiques, dans ce qu'il nomme un schéma tripartite de la phrase (Fig. 1) :

Speaker attitude / Force / Informational structure

Location of event relative to speech time

Internal properties of event

Fig. 1 (Carnie 2008)

7 La «boîte » intérieure représente le contenu propositionnel, ou plus techniquement les relations lexicales-thématiques de l'événement ou « état de choses " (state of affairs) décrit. Typiquement, dans la plupart des théories (voir infra pour P\&P), ce niveau sera représenté par quelque chose qui ressemblera de très près à un $\mathrm{VP}$, contenant le verbe et au moins l'un des participants (arguments) associés à l'événement. Du point de vue de la Grammaire Universelle dont se réclame la grammaire générative dans ses diverses variantes, ceci représente le noyau même de la proposition, et dans la plupart des théories on n'y trouvera que du matériau lexical.

8 Notons que tout syntagme est censé prendre la forme représentée en Fig. 2, avec une tête lexicale (nominale, verbale, adjectivale, prépositionnelle, adverbiale...) ou fonctionnelle ${ }^{2}$, qui va se projeter de la même façon. La Fig.2 détaille le mécanisme de cette projection, par première fusion (merge) avec un complément, puis par seconde fusion du constituant intermédiaire ainsi obtenu avec un spécifieur ${ }^{3}$ : 


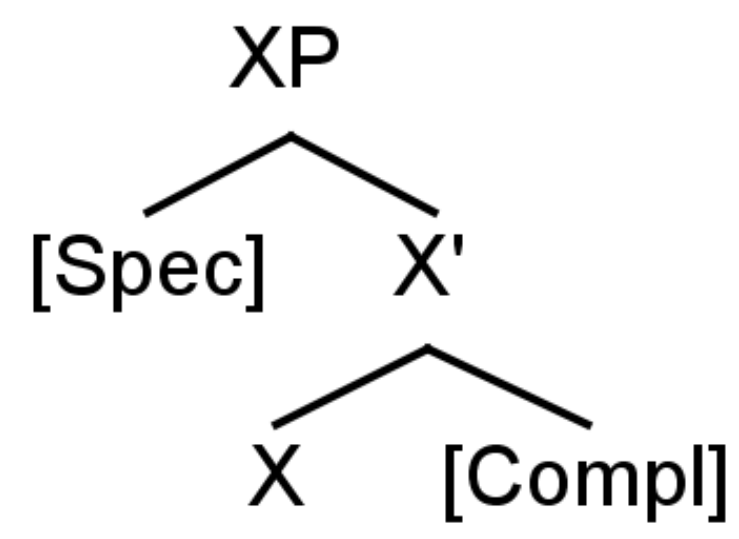

Fig. 2 : Le schéma X-barre

9 Le niveau intermédiaire de la Figure 1, quant à lui, représente la façon dont le schéma d'événement est mis en relation avec des marqueurs aspecto-temporels, qui vont le localiser par rapport à des repères, typiquement le présent de parole. Ce sont ici des éléments fonctionnels et non lexicaux que nous allons retrouver; et comme le fait remarquer Carnie (2008) ce niveau est "also associated with the notion of subjecthood"4. Car le sujet de surface de la proposition est le plus souvent analysé comme appartenant à cette couche (ou boîte)-là, ce qui au passage signifie que sa relation au verbe est bien plus distante que par exemple celle de l'objet direct quand ce dernier est présent.

Comme on peut le voir sur la Fig. 3, ce niveau est représenté en GG par une projection fonctionnelle I(nflexion), dont la tête va accueillir les marques verbales (réduites au seul présent simple sur l'exemple). Et effectivement, le sujet de surface de la phrase va se trouver dans la position [Spec, IP], qu'il y soit, selon les versions de la théorie, directement généré ou amené par un mouvement hors de la position [Spec, VP]. 


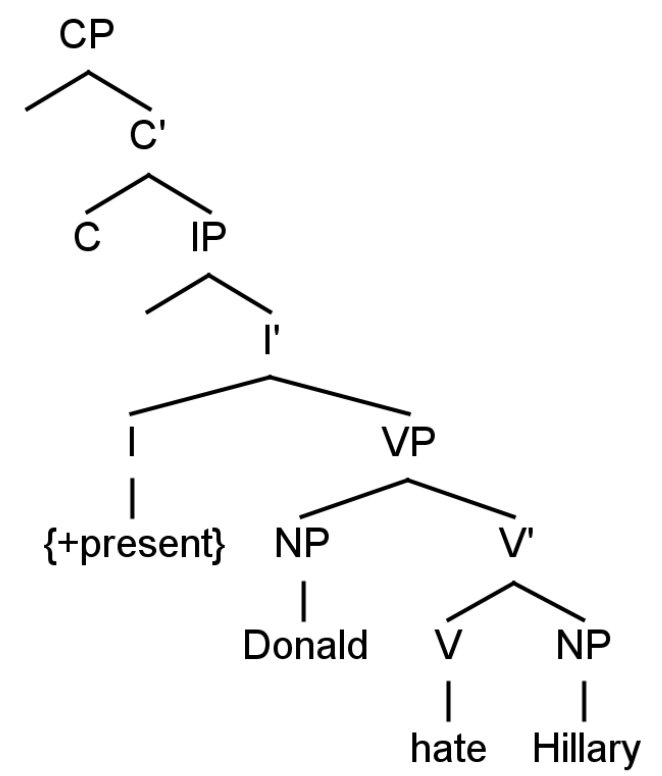

Fig. 3 (Donald hates Hillary)

11 Mais le plus intéressant pour notre propos se trouve plus haut dans la structure de la Figure 1. Nous disposons à présent, comme nous l'avons montré à propos de la prédication (Khalifa 2017), d'une prédication tensée. Et c'est la «boîte » extérieure qui va relier cette prédication aux attitudes du locuteur vis-à-vis de l'événement. C'est à nouveau du matériau fonctionnel que nous y retrouverons, donc, toujours pour citer Carnie (2008), "such notions as mood, focus, topic, and illocution"5.

C'est effectivement au troisième niveau de l'édifice (et donc dans la périphérie gauche de la phrase) que vont se jouer les choses en matière d'interrogation. Examinons donc de plus près ce qui s'y joue. Techniquement, il s'agit, comme on le sait, de la projection maximale de C ( = complémenteur, ou complementizer en version originale). Cette position est effectivement celle qui accueille, dans des cas d'enchâssement, les complémenteurs de l'anglais $\{T H A T, F O R$, WHETHER, IF\}. Le complémenteur est une catégorie fermée et donc fonctionnelle. Comme toute tête, elle sélectionne ses compléments, autrement dit, elle va sélectionner le type de IP, donc de phrase, qu'elle domine. Par exemple, THAT sélectionne une déclarative à temps fini, FOR une nonassertive infinitive, WHETHER une interrogative à temps fini ou non-fini, If une interrogative à temps fini ; les quatre sont donc en distribution complémentaire.

13 C'est ainsi par exemple que, si mon exemple inventé en Fig.3 était enchâssé, devenant ainsi complément d'un verbe recteur comme WONDER, on trouverait : 


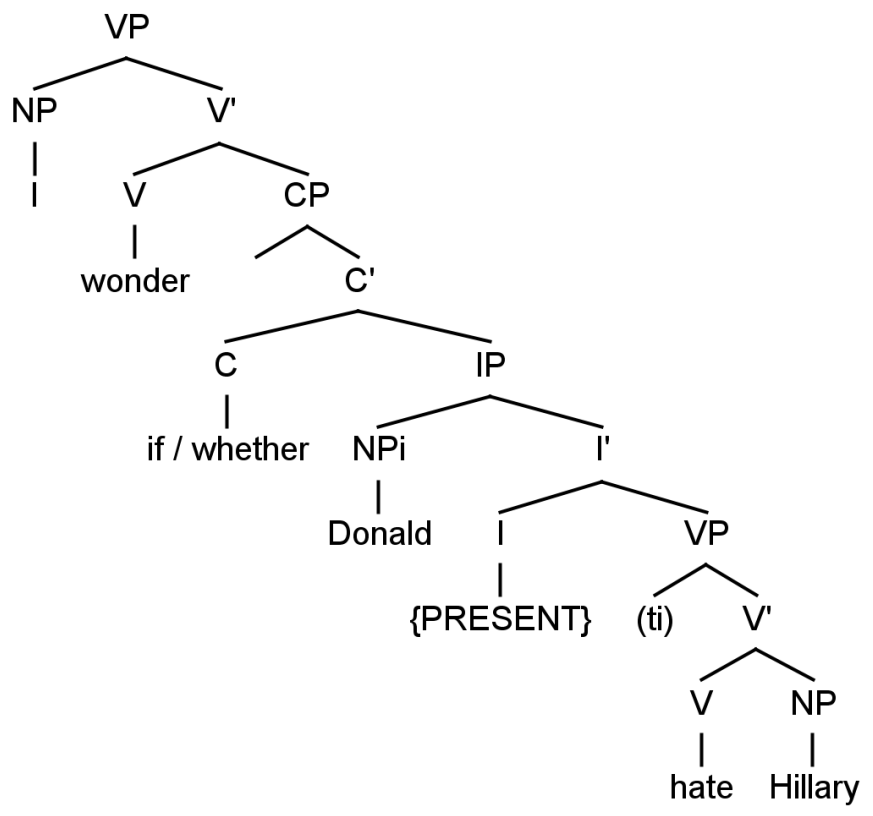

Fig. 3' (I wonder if/whether Donald hates Hillary)

\section{Les questions directes}

\subsection{Les questions fermées}

Mais laissons cela de côté pour l'instant, et voyons ce qui se passe en cas de question directe. Deux cas peuvent se présenter, comme il est banal de le constater, représentés par des exemples du texte comme : did Rosemary ever talk to you much? (1. 21), Did she ever talk about them ? (1.60), Were they intimate at all ? (1. 62), entre autres. Il s'agit là de ce que l'on nomme d'ordinaire questions fermées, yes/no questions, questions totales, etc. Les théories plus sémantiques analysent ce type comme remettant en cause l'ensemble de la prédication (être le cas / ne pas être le cas, p / p', etc.). Il est donc tout à fait naturel que l'opération en jeu porte sur le bloc prédicatif lui-même. Ce bloc, comme on l'aura compris des éléments présentés supra, est en GG représenté par le nœud I(nflection). Et c'est plus précisément le marqueur de temps qui va être concerné. Il va se déplacer et passer à gauche du sujet: c'est la fameuse "inversion sujetauxiliaire » dont parlent les manuels de grammaire, qui ont raison à une nuance près : le sujet, lui, reste dans sa position de sujet et ne se déplace pas ; comme on le voit sur la Fig. 4, c'est bel et bien le temps, en l'occurrence porté par l'auxiliaire $D 0^{6}$, qui va passer à gauche.

C'est le site d'arrivée, bien plus que le site de départ, qui est intéressant pour notre propos : car il n'est autre que la position $C$; on peut donc dire en première approche que complémenteurs et auxiliaires sont en distribution complémentaire. Mais on peut se souvenir également que cette position correspond à une couche qui, transthéoriquement, accueille des marqueurs renvoyant à l'attitude du locuteur vis-à- 
vis de la prédication. C'est la force illocutoire (cf. inter alii Huddleston 2002) de l'énoncé (interrogatif en l'occurrence) qui va être ainsi marquée. Cette force illocutoire, de façon plus technique, est matérialisée sur le diagramme par la présence d'un trait \{+interrogatif\}. Ici, je l'ai symbolisé par WH, mais on peut tout aussi bien le symboliser par un point d'interrogation (ou par ce qu'on voudra). Ce qui compte, c'est qu'il s'agit d'un trait abstrait, en distribution complémentaire avec des morphèmes libres. On pourra ainsi symboliser par тHAT le trait \{+ assertif, + déclaratif\}, et on sait que des morphèmes comme that peuvent parfaitement être obligatoires dans certaines langues pour marquer les déclaratives (voir le cas bien connu du que du béarnais, où l'on aura par exemple que cante pour « il/elle chante », cf. Joly 1976).

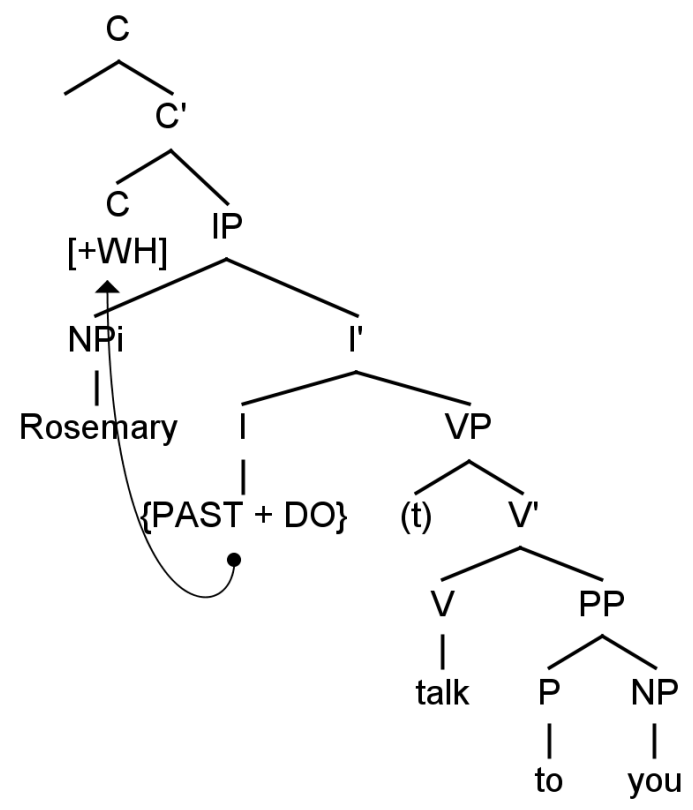

Fig. 4 (Did Rosemary talk to you ?)

\subsection{Les questions ouvertes}

Le second cas est celui représenté par bon nombre d'exemples du texte, dont When had that begun? What was the cause of it? (1. 2), ou encore when exactly had his abstraction become something more than natural ? (1. 4) ou How intimate was she with them ? (1. 41), What did Sandra Farraday think about it? (1. 68). Il s'agit traditionnellement de questions partielles, questions ouvertes, ou wH-questions. Dans ce cas-là, on va retrouver un second mouvement, le mouvement-wH.

17 Ce mouvement consiste à faire sortir un mot-WH, ou un syntagme comportant un motWH, de son site d'origine, pour l'amener, linéairement, en "tête de phrase ", dans la position la plus à gauche. Techniquement, le site d'arrivée sera le spécifieur de $\mathbf{C P}$, donc toujours dans le domaine du complémenteur. Nous illustrons ce phénomène avec les Fig. 5 et 6, qui correspondent respectivement à des cas où $w H$ est argument du verbe (plus exactement «attribut du sujet » dans une optique traditionnelle) et circonstant 
ou adjoint du verbe. On pourrait ajouter que l'exemple de la 1. 41, How intimate was she with them ?, illustre deux phénomènes; l'un est assez banal, c'est l'appartenance, en dépit de la morphologie apparente, de How au paradigme fermé des mots-WH. Le second mérite que l'on s'y arrête brièvement. On constate en effet que le mot-wH ne s'est pas déplacé tout seul, mais qu'il a entraîné l'adjectif avec lui. On vérifiera en effet que le site de départ est celui d'adverbe de degré dudit adjectif ${ }^{7}$ (she was DEG intimate with them), et le mouvement nous donne bien :

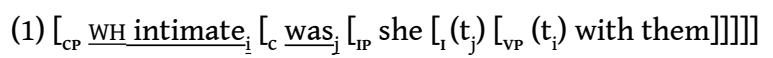

et non, en l'occurrence, ${ }^{*}$ How was she intimate with them ?, où l'adjectif serait resté in situ. Le phénomène d'entrainement de tout ou partie d'un syntagme par un mot-wH dans son déplacement porte en grammaire générative le joli nom de pied-piping ${ }^{8}$. Il est le plus fréquent avec des prépositions (nous n'en avions malheureusement pas d'exemple dans le texte), comme With what did she wipe his feet? With what did she anoint them ?, et pose une multitude de questions, comme le caractère obligatoire ou facultatif, et le contraste avec le cas inverse, que l'on nomme stranding ou orphelinage (What did she wipe his feet with ? etc.). Ici, nous sommes dans un cas de pied-piping obligatoire.

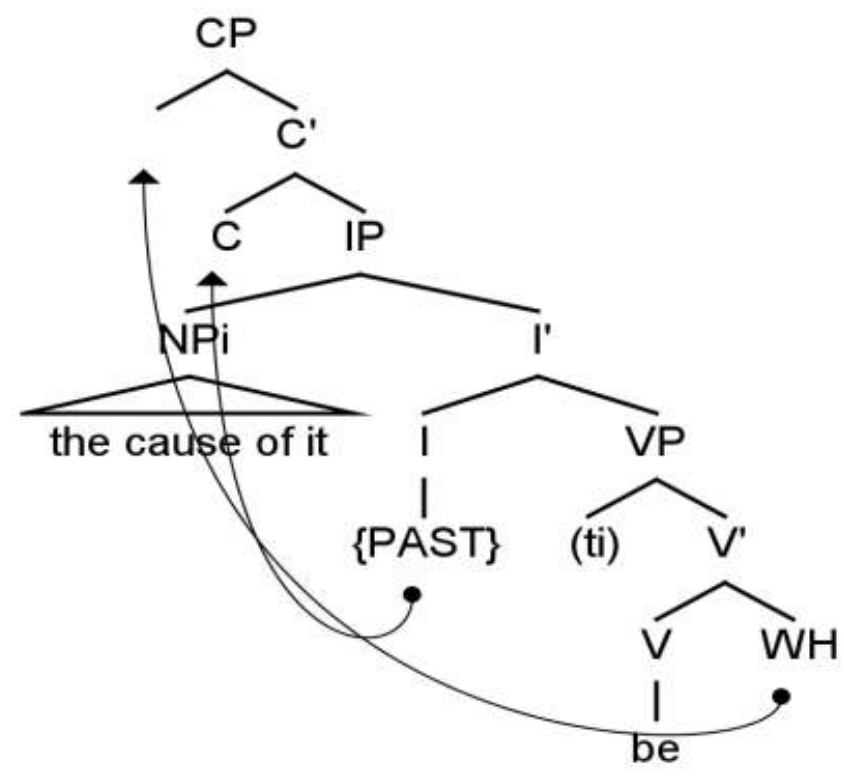

Fig. 5 (What was the cause of it ?) 


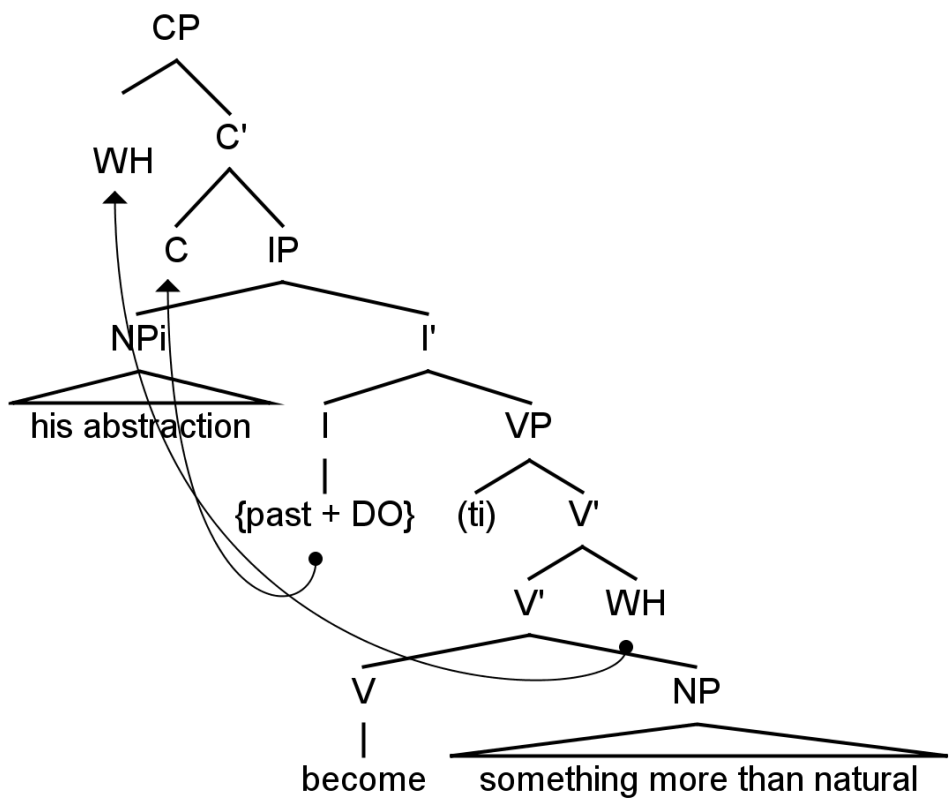

Fig. 6 (When did his abstraction become... ?)

(2) This cot was guarded by a romantic population, with one or two of whose units Michael could willingly have dispensed : one bar in particular, set very much askew, seemed sly and malignant. (Compton McKenzie, Sinister Street)

Quelques remarques sur le mouvement-wH. Tout d'abord, il est des langues qui n'en ont pas du tout (comme le japonais), et il est des langues comme le français où, même si le mouvement existe, il est à présent devenu rare à l'oral, où le mot-WH reste le plus souvent in situ (« t'es allé où ?», « elle a dit quoi ? ", etc.). L'anglais connaitt également l'interrogatif in situ, mais dans une proportion bien moindre. On en a un exemple, mais tronqué, dans notre texte, à la 1.23 , About what?, qui ne comporte pas de verbe. Cela n'est bien évidemment pas à confondre avec un exemple comme le tout dernier du texte, 1. 98:...he was more or less of a casual acquaintance?, où il n'y a aucune marque d'interrogation à part le point d'interrogation, qui correspondrait à une courbe intonative particulière à l'oral. La grammaire générative n'a, somme toute, rien d'autre à dire de tels exemples que de constater qu'ils ont la syntaxe de l'affirmative...

21 Seconde remarque, le mouvement- $w H$ peut parfaitement franchir plus d'une frontière de phrase en cas d'enchâssement. On peut illustrer cela en manipulant quelque peu l'exemple de la 1. 26: She thought she saw what was in his mind'. Si nous faisons franchir à what les frontières du CP de l'imbriquée, on peut obtenir :

(3) What ${ }_{i}$ did she think $\left[_{C P}\left(t_{i}\right)\right.$ she saw $\left(t_{i}\right)$ was in his mind] ?

22 On peut, à partir d'exemples fabriqués, montrer que le phénomène est récursif et ne semble pas avoir d'autres limites que celle de l'intelligibilité de la phrase :

(4a) What ${ }_{i}$ was he reading $\left(t_{i}\right)$ ?

(4b) What ${ }_{i}$ did he say $\left[{ }_{\mathrm{CP}}\right.$ that he was reading $\left.\left(\mathrm{t}_{\mathrm{i}}\right)\right]$ ? 
(4c) What ${ }_{\mathrm{i}}$ does she believe $\left[\mathrm{cP}\right.$ that he said $\left[\mathrm{cP}\right.$ that he was reading $\left.\left.\left(\mathrm{t}_{\mathrm{i}}\right)\right]\right]$ ?

(4d) What ${ }_{\mathrm{i}}$ do they claim $\left[_{\mathrm{CP}}\right.$ that she believes $\left[_{\mathrm{CP}}\right.$ that he said $\left[_{\mathrm{CP}}\right.$ that he was reading

$\left.\left.\left(\mathrm{t}_{\mathrm{i}}\right)\right]\right]$ ?

Mais en fait, en dépit de ces données, il existe des limites et des contraintes, identifiées en GG depuis les travaux de Ross (1967) ; depuis lors et jusqu'à ce jour est parue une masse considérable d'études sur les phénomènes que l'on appelle islands ou "îlots", jolie métaphore pour signifier que les mots-WH ne peuvent pas en sortir (en être « extraits »). Un exemple entre mille : les complétives de nom forment de tels îlots :

(5a) He made the claim that he had met Rosemary

(5b) * Who ${ }_{i}$ did he make the claim that he had met $\left(\mathrm{t}_{\mathrm{i}}\right)$ ?

\section{Les questions imbriquées}

En fait, ces exemples, avec le THAT qui réapparaît, ou qui peut réapparaître, vont surtout nous servir de transition vers le dernier type que nous souhaitons étudier, illustré par des exemples comme Another day he asked her suddenly who Rosemary's best women friends had been (1.37-38), ou encore, mais avec des différences significatives, ...to her question as to what was the matter, he replied briefly, 'Nothing.' (1. 14).

Le premier de ces exemples illustre un cas tout à fait classique, mais qui a suscité, au fil des années, des quantités considérables d'analyses et de commentaires, et ce dans toutes les théories linguistiques. Regardons donc du côté de la question directe : en simplifiant les choses pour ne pas alourdir la représentation inutilement, on peut la réduire à Who were Rosemary's best women friends?, autrement dit :

(6a) $\left[{ }_{C P}\right.$ who ${ }_{i}\left[{ }_{C}\right.$ were ${ }_{j}\left[\right.$ IP Rosemary's best women friends $\left.\left.\left.\left(t_{j}\right)\left(t_{i}\right)\right]\right]\right]$

Ou encore, sur une représentation arborescente, qui finalement dit la même chose, mais en deux dimensions : 


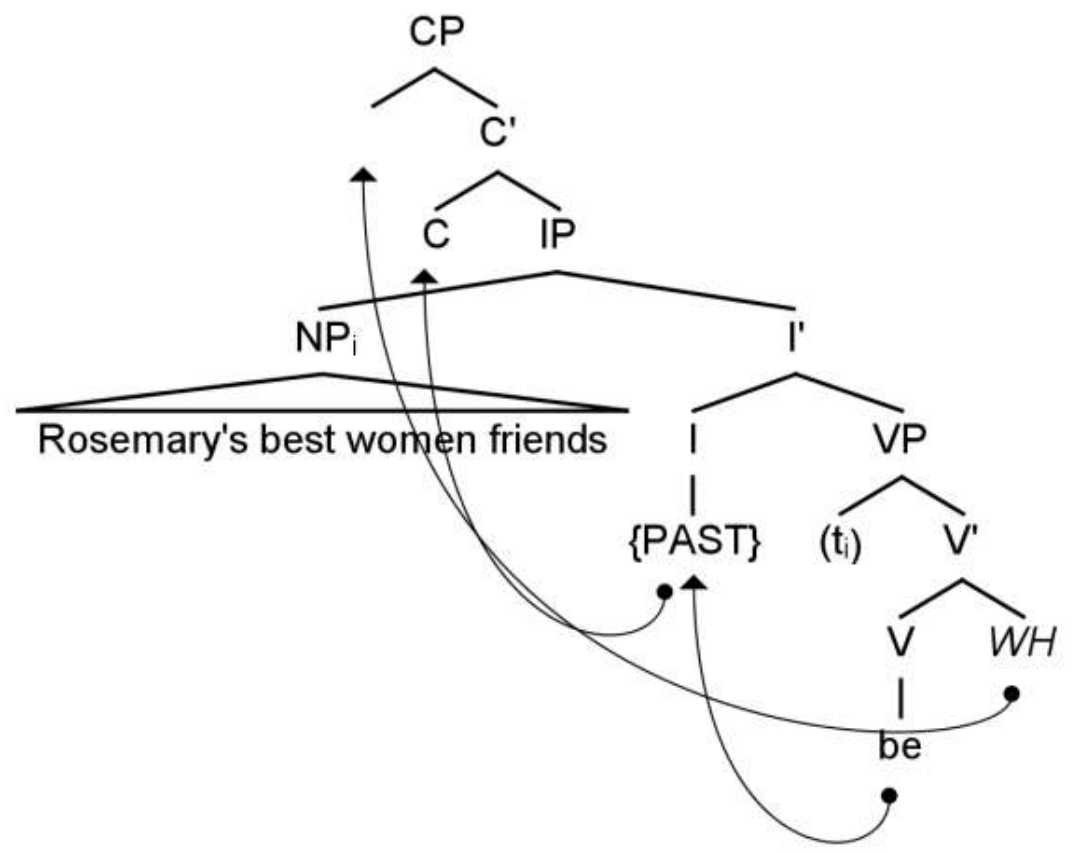

Fig. 7 (Who were Rosemary's best friends ?) plus haut, à savoir d'une part le I-TO-c movement (le verbe copule, qui porte la marque de temps, est bien monté dans la position C), et d'autre part le mouvement-wH, qui va sortir le mot-WH de son site d'origine (en l'occurrence ici, une position d'attribut du sujet, puisqu'il s'agit de $B E$ copule, mais en tout cas une position argumentale, sœur du verbe), pour l'amener très classiquement dans la position de spécifieur de $\mathrm{CP}$. Mais ce que nous n'avons pas précisé plus haut, c'est que les deux mouvements sont indépendants l'un de l'autre. On peut avoir, bien évidemment, I-TO-C movement sans mouvement-WH, ce qui est classiquement le cas des questions fermées, mais on peut également avoir mouvement-wH sans déplacement de l'inflexion. C'est par exemple ce qui va normalement se passer en anglais standard dans une question imbriquée (les traditionnelles « interrogatives indirectes ») :

(7b) $\left[_{\mathrm{V}}\right.$ ask $\left[{ }_{\mathrm{CP}}\right.$ who ${ }_{\mathrm{i}}\left[_{\mathrm{C}}\right.$ RP Rosemary's best women friends were $\left.\left.\left.\left.\left(\mathrm{t}_{\mathrm{i}}\right)\right]\right]\right]\right]$

La question qui bien évidemment doit être posée est celle de l'absence de I-TO-C movement dans les questions imbriquées. Reprenons la représentation supra en Fig. 3'. Une constatation simple : la présence d'un complémenteur explicite (IF Ou WHETHER) sous C bloque tout mouvement de I vers cette position. Mais qu'en est-il dans les questions imbriquées ouvertes, où apparemment cette même position C est vide ?

L'analyse standard (cf. Chomsky \& Lasnik 1977) est que toute subordonnée (toute complétive, en d'autres termes), suppose un complémenteur sous C. Ce complémenteur, sous certaines conditions, est parfois effacé, ce qui ne veut pas dire que la position est vide, mais qu'il est phonologiquement nul (unpronounced). Le phénomène est assez banal avec le complémenteur FoR, parfois présent, parfois effacé, en fonction des verbes recteurs ou des dialectes : 
(8a) They wanted (for) her to break free of her family, which they thought were monsters and become a new person.

(8b) They wanted to ask, but they preferred for her to receive a well-deserved rest from a very trying battle.

Dans l'optique de la GG, cette analyse est à généraliser à tous les complémenteurs, et en particulier à THAT, qui, rappelons-le, sélectionne une proposition (IP) assertive à temps fini ; c'est ainsi que, si nous manipulons un peu notre phrase en Fig 3', nous obtenons :

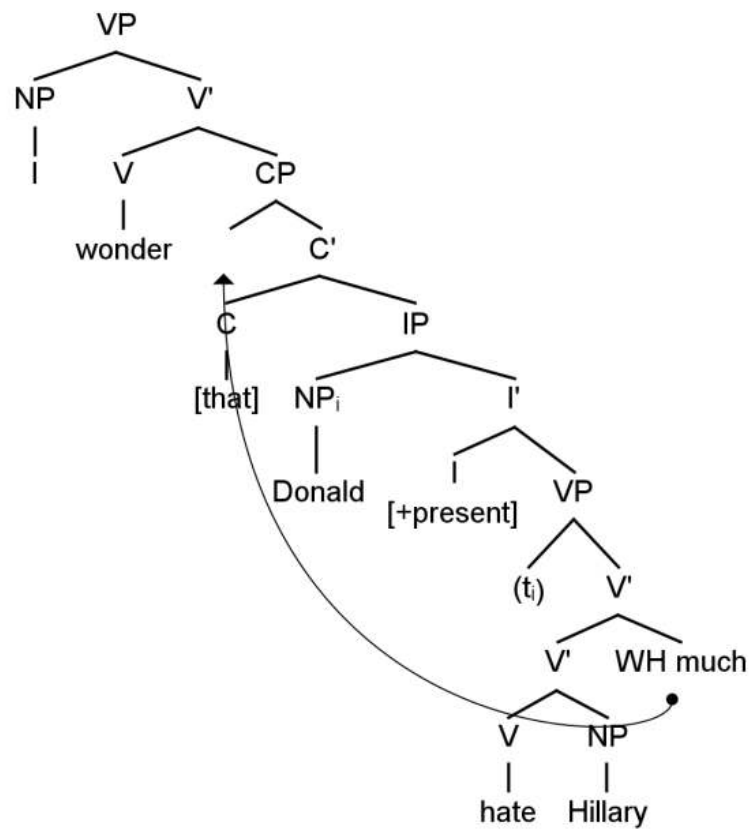

Fig. 3" (I wonder how much [that] Donald hates Hillary)

L'une des raisons décisives pour lesquelles on a pu postuler une structure comme celle de la Fig. 3" est que, même si en anglais standard contemporain, le complémenteur ne serait pas prononcé, la coexistence d'un mot ou d'une expression WH- avec THAT est très largement attestée, d'abord historiquement ${ }^{10}$ :

(9a) I wolde fayn knowe how that ye understonde thilke wordes and what is youre sentence

'I would like to know how you understand these same words'

(9b) And forther over, it is necessarie to understonde whennes that synnes spryngen, and how they encreessen

'And moreover, it is necessary to understand where sins come from, and how they increase'

(9c) Now shal ye understonde in what manere that synne wexeth or encreesseth in man.

'Now you shall understand in what manner sin grows or increases in man.' de A. Henry (Henry 1995) qu'il s'agit là d'un des traits caractéristiques de l'angloirlandais, et plus spécialement de sa variété Belfast English :

(10 a) I wonder which dish that they picked.

(10b) They didn't know which model that we had discussed. 
standard, on trouve assez couramment des exemples comme :

(11a) I realized whatever else that happened, I didn't want to die.

(overheard at $19^{\text {th }}$ and Walnut Streets, 23 May 2015)

(11b) ....and it helps us understand what a profound Mystery that fear really is, like

love. (Jeff Golliher. 2011. Moving Through Fear. Tarcher. 12.)

(11c) It's amazing, how much that they learned.

(overheard at $19^{\text {th }}$ and Walnut Streets, 28 March 2015)

(11d) I didn't know how dishonest that I was.

(overheard at $19^{\text {th }}$ and Walnut Streets, 7 March 2015)

(11e) You could come and see which one that you're interested in.

(Mormon missionary, in conversation, International Creche Exhibit, Mormon

Temple, Washington, DC, 25 December 2014)

(11f) I don't know why that you're right.

(J T, in conversation, 16 December 2014 ; no marked intonation on why)

On sait que l'on retrouve le même phénomène dans bon nombre de langues, $\mathrm{y}$ compris en français populaire : À qui que tu veux parler? Où que tu vas ? On sait pas où qu'elle habite. Le gars avec qui que je travaille (ce dernier exemple, et quelques autres précédents, montrant bien que le phénomène se retrouve dans d'autres cas impliquant un mouvement- $W H$, comme les relatives ou les exclamatives). Notons que ce qui est en revanche totalement prohibé, et ce dans tous les dialectes, c'est, comme en (12b) cidessous, la co-occurrence d'un complémenteur explicite et d'un auxiliaire monté par mouvement I-TO-C :

(12a) \%It's gonna ask you [do you wanna make a transfer].

(12b) *It's gonna ask you [if do you wanna make a transfer].

Ce qui est, dans l'optique qui est la nôtre, extrêmement facile à comprendre : nous avons dit supra que complémenteurs et auxiliaires étaient en distribution complémentaire, puisqu'ils sont en quelque sorte en concurrence pour la même position C. Et, a contrario, il faut qu'une des deux positions soit occupée, ce qui prohibe, dans tous les dialectes, des exemples comme :

(12c) *It's gonna ask you [ - you wanna make a transfer].

37 Tout cela n'empêche évidemment pas de trouver, et en abondance, des cas où coexistent mouvement-WH et I- TO-C movement dans les questions imbriquées. On retrouve encore ce cas de figure de façon régulière en anglo-irlandais, mais également dans un certain nombre de variétés d'américain :

(13a) She asked who had I seen.

(13b) They wondered what had John done.

(13c) They couldn't understand how had she had time to get her hair done.

(13d) I wondered where were they going. (Henry 1995, 106 ; 116)

(14) I don't know what color are we, but it doesn't matter.

Notons d'ailleurs au passage que l'on peut également trouver, dans ces dialectes, le même phénomène associé à un mouvement- $W H$ long, comme dans :

(15) Who did John hope would he see?

Ce genre d'exemple peut vraisemblablement s'expliquer par un phénomène de parataxe : les imbriquées ne sont sans doute justement pas ressenties comme telles, ce qui explique la syntaxe avec I-TO-C movement. Est-ce ce qui se passe avec notre dernier exemple, ...to her question as to what was the matter, he replied briefly, 'Nothing.' (1. 14) ? Ici, AS TO est à analyser comme une préposition complexe (elle occupe, dans une étude 
classique (Fang 2000) le $42^{\mathrm{e}}$ rang dans le classement des prépositions les plus fréquentes en anglais). Et la structure, globalement, est à analyser comme une complétive de nom, et non comme une interrogative indirecte. Ce qui doit tout de même un peu changer la donne ; par curiosité, nous avons, à défaut d'une véritable étude de corpus, fait un petit sondage Google, que l'on trouvera ci-dessous en Fig. $8^{11}$ : syntaxe et de la pragmatique : la parataxe doit jouer un rôle majeur pour effacer en quelque sorte l'imbrication et en revenir à une question directe : ...her question: "what is the matter?". Cela se conjugue avec le figement de "What's the matter?", que l'on a vérifié en testant de la même façon, et avec les mêmes résultats, what the subject was / what was the subject, what the problem was / what was the problem, et avec des résultats bien moins probants sur des formes moins figées, comme who the man was / who was the man.

\section{Conclusion}

51 Nous conclurons en évoquant brièvement des développements plus récents de la théorie, initiés en particulier par Rizzi (1997). Alors que CP a longtemps été analysé comme une seule projection avec une tête $\mathrm{C}$ et une position de spécifieur SpecCP, Rizzi montre qu'il doit être conçu comme un domaine regroupant un ensemble de projections. Il a proposé la structure suivante (16), où Force encode la valeur illocutoire et Fin (Finitude) détermine si la proposition qui suit doit être finie ou non-finie. Entre les deux se trouvent des positions de Focus et de Topique; ce dernier pouvant être itératif, en tout cas en italien. Si aucun élément outre le complémenteur n'est présent dans la périphérie gauche, on dit que le CP n'est pas éclaté.

(16) [.. Force [.. Topic [.. Focus [.. Topic [.. Finiteness [IP ...

Credo che ieri QUESTO a Gianni avreste dovuto dirgli

'I believe that yesterday THIS to Gianni you should have said'

La Fig. 9 ci-dessous dit exactement la même chose en deux dimensions; l'intérêt de cette approche est d'intégrer dans la syntaxe des dimensions pragmatiques qui lui faisaient cruellement défaut, et au passage de pouvoir fournir une analyse et une représentation adéquates à des contrastes ${ }^{12}$ comme : 


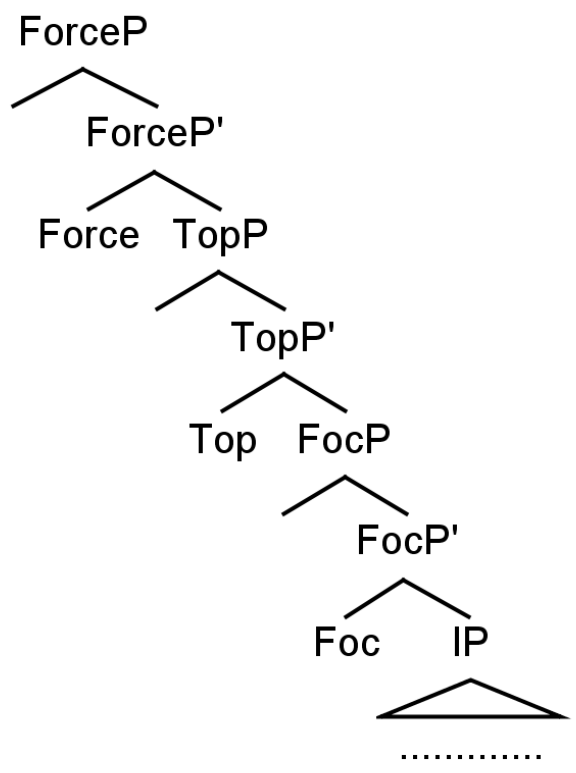

Fig. 9 Split-CP hypothesis

(17) a. I cannot see how, this kind of behavior, we can tolerate in a civilized society

b. *I cannot see, this kind of behavior, how we can tolerate in a civilized society

(18) a. On the table, which dishes are you going to put?

b. *Which dishes are, on the table, you going to put?

53 Ce qui, au passage, permettra de montrer que les mots- $W H$, qui sous l'ancienne représentation occupaient toujours la même position Spec, CP, vont se comporter différemment dès lors qu'un topique ou un focus est inséré. Par exemple dans la question imbriquée en (17), où par ailleurs le NP this kind of behaviour est sans conteste un topique, on voit par le contraste entre (17a) et (17b) que how ne peut qu'occuper une position inférieure à ce dernier (ou à sa droite, si l'on raisonne en deux dimensions, $c f$. (16)). En revanche, dans la question directe en (18), où on the table est aussi un topique, on constate que l'interrogatif ne peut qu'être à sa gauche (dans une position plus haute). Le lecteur curieux comprendra que la seule position disponible dans le premier cas est le spécifieur de ForceP, et dans le second cas celui de FocP. On peut dès lors avancer l'hypothèse que les mots-WH ont cette propriété très particulière de pouvoir soit marquer le focus quand ils sont interrogatifs (donc quand ils sont une demande d'information nouvelle), soit marquer seulement la force illocutoire de la phrase en cas d'imbrication ${ }^{13}$. 


\section{BIBLIOGRAPHIE}

Carnie, A. (2008). Constituent Structure. Oxford : Oxford University Press.

Chomsky, N. (1965). Aspects of the Theory of Syntax. Cambridge (Mass), MIT Press.

Chomsky, N. \& Lasnik H. (1977). 'Filters and Control'. Linguistic Inquiry, 8 (3), pp. 425-504.

Culicover, P. (1997). Principles and Parameters : An Introduction to Syntactic Theory. Oxford : Oxford University Press.

Fang, A. (2000). 'A lexicalist approach towards the automatic determination for the syntactic functions of prepositional phrases'. Natural Language Engineering, 6 :183-201.

Halliday, M.A.K. (1985). Introduction to Functional Grammar. London : Arnold.

Henry, A. (1995). Belfast English and Standard English. Oxford : Oxford University Press.

Huddleston, R. (2002). ‘Clause type and illocutionary force'. In : Huddleston, R. \& Pullum, G. (dirs.) The Cambridge Grammar of the English Language. Cambridge : Cambridge University Press. pp. 851-945.

Jackendoff, R. (1977). X-bar Syntax. Cambridge (Mass.) : MIT Press.

Joly, A. (1976). « 'Que’ et les autres morphèmes énonciatifs du béarnais : essai de psychosystématique ». Actes du $13^{e}$ Congrès de Linguistique et Philologie Romanes, Québec: Presses de l'Université Laval, vol. 1, p. 411-433.

khalifa, J.-C. (2014). 'Syntax and Degree'. Publications en ligne de l'ALAES (http://bv.ubordeaux3.fr/pub/docs/group-degre/Khalifa_SyntaxAndDegree.pdf)

Khalifa J.-C. (2017 - à paraître). « La prédication en grammaire générative ». In Vincent-Durroux L. (éd.), Prédication et prépositions en anglais : la mise en relation au prisme de différentes approches linguistiques, CORELA volume hors-série (https://corela.revues.org/)

Pollock, J.-Y. (1992). « Opérateurs nuls, dont, questions indirectes, et théorie de la quantification ». In L. Tasmowski \& A. Zribi-Hertz (eds.), Hommages à Nicolas Ruwet. Gand, Communication \& Cognition.

Radford, A. (2009). Analysing Sentences : A Minimalist Approach. Cambridge : Cambridge University Press.

Rizzi, L. (1997). ‘The Fine Structure of the Left Periphery'. In Haegeman L. (ed.), Elements of Grammar, Dordrecht : Kluwer.

Ross, J. (1967). Constraints on Variables in Syntax, Thèse, M.I.T. (publiée sous le titre Infinite Syntax !. New York : Ablex Publishing Corporation, 1986)

\section{NOTES}

1. Nous utilisons «événement » dans ce sens vague ici, comme l'anglais utilise state of affairs. Ce terme se veut neutre au plan théorique, et subsume états / processus etc.

2. Jackendoff (1977), qui introduit la théorie X-barre, n'utilisait pas encore les têtes fonctionnelles (par exemple I(nflection), $\mathrm{C}$ (omplementizer), etc.), qui sont introduites dans les diverses versions de la théorie à compter des années 80 . Mais même lorsque la tête est 
fonctionnelle, l'architecture est postulée identique, avec les deux fusions successives (Xcomplément et X'-spécifieur) pour donner le niveau syntagmatique (XP).

3. Deux remarques au passage : «complément » et "spécifieur » sont des positions structurales, et sont optionnelles, au sens où elles peuvent ne pas être instanciées.

4. Carnie, op. cit. p. 221.

5. Carnie, op. cit. p. 221.

6. Nous ne rouvrirons pas ici le débat sur $D O$; les générativistes le considèrent généralement comme un morphème à valeur uniquement syntaxique et parlent de Do-support. Sur la représentation de la Fig.4, nous l'avons généré directement dans la position $\mathrm{I}$, où il va fusionner avec la marque de temps fini. Mais en toute orthodoxie, il nous faudrait dire qu'il est injecté directement dans la position d'arrivée sous $\mathrm{C}$.

7. On admet que la catégorie Degré est la catégorie fonctionnelle qui domine l'adjectif, comme le déterminant est la catégorie fonctionnelle qui domine le nom, ou plus évidemment encore, l'inflexion la catégorie fonctionnelle qui domine le verbe. Sur ce point, nous renvoyons à Khalifa 2014.

8. J.-Y. Pollock (1992) avait proposé en français le non moins joli terme de « hamelinage ». Notons tout de même que Pollock lui-même disait tenir ce terme d'Alex Grosu.

9. Au passage, l'énoncé original n'est pas pour nous une interrogative indirecte, mais une relative nominale.

10. Les exemples en (9) sont tous extraits de The Parson's Tale (c1390), Manuscript Ellesmere $26 \mathrm{C}$ 9, Huntington Library, San Marino, CA.

11. Les noms-têtes, à première vue, sont en majorité écrasante DOUBT et QUESTION.

12. Empruntés à Radford 2009; nous renvoyons le lecteur intéressé au chapitre 8 ('Split Projections) de cet ouvrage pour une excellente synthèse.

13. Si l'on étendait l'analyse, on s'apercevrait d'ailleurs que les $W H$ relatifs, qui précisément ne constituent jamais une demande d'information nouvelle, se comportent exactement, soumis aux mêmes tests, comme dans les interrogatives indirectes et vont occuper une position sous ForceP.

\section{RÉSUMÉS}

Dans cette contribution, nous examinons, dans la perspective de la grammaire générative version Principles \& Parameters, l'architecture des interrogatives, en traitant successivement des questions directes ouvertes et fermées, puis des questions imbriquées, après de brèves considérations théoriques permettant de saisir les niveaux de construction et les différents mouvements (I-to-C movement, WH-movement) mis en jeu. Nous concluons par un très bref aperçu des mêmes structures analysées dans la perspective un peu plus récente du Split-CP, où les projections sont multipliées dans la périphérie gauche des phrases.

In this chapter, we address the generative approach to interrogative structures, mostly under the Principles \& Parameters framework. After a brief theoretical overview, we discuss direct open and closed questions, then embedded questions, showing how I-to-C movement and WH-movement come into play. We then conclude on very brief considerations on more recent developments about the 'left periphery' of sentences, involving splitting the CP projection. 
INDEX

Mots-clés : Questions, mouvement-WH, complémenteur, périphérie gauche, grammaire générative

Keywords : Questions, WH-movement, complementizer, left periphery, generative grammar

\section{AUTEUR}

JEAN-CHARLES KHALIFA

Université de Poitiers

Forell-Cerlitep (EA 3816)

jean.charles.khalifa@univ-poitiers.fr 